\title{
PERIODIC ORBITS AND NON-INTEGRABILITY OF HÉNON-HEILES SYSTEMS
}

\author{
JAUME LLIBRE ${ }^{1}$ AND LIDIA JIMÉNEZ-LARA ${ }^{2}$
}

\begin{abstract}
We apply the averaging theory of second order to study the periodic orbits for a generalized Hénon-Heiles system with two parameters, which contains the classical Hénon-Heiles system. Two main results are shown.

The first result provides sufficient conditions on the two parameters of these generalized systems, which guarantee that at any positive energy level, the Hamiltonian system has periodic orbits. These periodic orbits form in the whole phase space a continuous family of periodic orbits parameterized by the energy.

The second result shows that for the non-integrable Hénon-Heiles systems in the sense of Liouville-Arnol'd, which have the periodic orbits analytically found with averaging theory, cannot exist any second first integral of class $\mathcal{C}^{1}$. In particular, we prove that the classical Hénon-Heiles system and many generalizations of it are not integrable in the sense of Liouville-Arnol'd for any second first integral of class $\mathcal{C}^{1}$.

Moreover the tools we use for studying the periodic orbits and the non LiouvilleArnol'd integrability, can be applied to Hamiltonian systems with an arbitrary number of degrees of freedom.
\end{abstract}

\section{INTRODUCTION}

The classical Hénon-Heiles potential consist of a two dimensional harmonic potential plus two cubic terms. It was introduced in 1964, as a model for studying the existence of a third integral of motion of a star in an rotating meridian plane of a galaxy in the neighborhood of a circular orbit [15]. The classical Hénon-Heiles potential has been generalized by introducing two parameters to each cubic term

$$
\frac{1}{2}\left(p_{x}^{2}+p_{y}^{2}+x^{2}+y^{2}\right)+B x y^{2}+\frac{1}{3} A x^{3} .
$$

such that $B \neq 0$, with $x, y, p_{x}, p_{y} \in \mathbb{R}$. Then the classical Hénon-Heiles Hamiltonian system corresponds to $A=-1, B=1$. The Hamiltonian system is given by

$$
\begin{aligned}
& \dot{x}=p_{x}, \\
& \dot{p}_{x}=-x-\left(A x^{2}+B y^{2}\right), \\
& \dot{y}=p_{y}, \\
& \dot{p}_{y}=-y-2 B x y .
\end{aligned}
$$

As usual the dot denotes derivative with respect to the independent variable $t \in \mathbb{R}$, the time. We name (2) the Hénon-Heiles Hamiltonian systems with two parameters, or simply the Hénon-Heiles systems.

The periodic orbits are the most simple non-trivial solutions of a differential system. Their study is of particular interest because the motion in their neighborhood can be determined by their kind of stability. Furthermore, if the system is non-integrable in the sense of Liouville -Arnol'd, the existence of isolated periodic orbits in the energy

Key words and phrases. periodic orbits, integrability, Hénon-Heiles, averaging theory. 
levels of a Hamiltonian system with multipliers different from 1 is related with the non existence of any second first integral of class $\mathcal{C}^{1}$, so the study of these kind of periodic orbits becomes relevant.

The periodic orbits in the Hénon-Heiles potential have been numerically studied and classified by Churchil et. al. [10], Davies et. al. [11] and others [5, 12, 26]. Maciejewski et. al. [19] did an analytical study of a more general Hénon-Heiles Hamiltonians including a third cubic term of the form $C x^{2} y$, which can be removed by a proper rotation, and two more parameters associated with the quadratic part of the potential. They proved the existence of connected branches of non-stationary periodic orbits in the neighborhood of a given degenerate stationary point.

In this work we use the averaging method of second order to compute periodic orbits as it is established by Buică and Llibre [6], see section 2 for a summary of this method. This method allows to find periodic orbits of the Hénon-Heiles systems (2), up to first order in $\epsilon$, at any positive values of the energy as a function of the parameters $A$ and $B$. Roughly speaking, this method reduces the problem of finding periodic solutions of some differential system to the one of finding zeros of some convenient finite dimensional function. Our main result on the periodic orbits of the Hénon-Heiles system (2) is summarized as follows.

Theorem 1. At every positive energy level the Hénon-Heiles Hamiltonian system (2) has at least

(a) one periodic orbit if $(2 B-5 A)(2 B-A)<0$ (see Figure 1$)$,

(b) two periodic orbits if $A+B=0$ and $A \neq 0$ (this case contains the classical Hénon-Heiles system), and

(c) three periodic orbits if $B(2 B-5 A)>0$ and $A+B \neq 0$ (see Figure 2).

Theorem 1 is proved in section 3 .

It is well known that integrable and non-integrable Hamiltonian systems can have infinitely many periodic orbits. However it is difficult to find a whole family of periodic orbits in an analytical way, specially if the Hamiltonian system is non-integrable. Here we find them up to first order in $\epsilon$. Once we have shown that at any positive energy level there exist periodic orbits, we can use these particular periodic orbits to prove our second main result about the non-integrability in the sense of Liouville-Arnol'd of Hénon-Heiles systems (2).

Theorem 2. Assume that the Hénon-Heiles Hamiltonian system (2) satisfies the assumptions of one of the statements of Theorem 1, and denote by $(\mathrm{x})$ this statement. Then, under the assumption of statement (x),

(a) either the Hénon-Heiles Hamiltonian system is Liouville-Arnol'd integrable and the gradients of the two constants of motion are linearly dependent on some points of the periodic orbits found in statement (x) of Theorem 1,

(b) or the Hénon-Heiles system is not Liouville-Arnol'd integrable with any second first integral of class $\mathcal{C}^{1}$.

Theorem 2 is proved in section 4 . For a precise definition of the notion of integrability in the sense of Liouville-Arnol'd, see again section 4. From now on we shall use the definition of integrability in the sense of Liouville-Arnol'd.

The following corollary is proved at the end of section 4 . 
Corollary 3. At every positive energy level such that $B(2 B-A)>0, A-B \neq 0$ and $2 B-5 A \neq 0$, the Hénon-Heiles Hamiltonian system is not Liouville-Arnol'd integrable, with any second first integral of class $\mathcal{C}^{1}$.

Before the present work many different results on the integrability and non-integrability of the Hénon-Heiles Hamiltonian systems (2) have been proved, or on other more general Hénon-Heiles Hamiltonians as for instance the work of Grammaticos, Dorizzi and Padjen [14]. The case $A=B$ was explicitly integrated by Chazy [7] as the sum of two Weierstrass functions, and the case $A-6 B=0$ is integrable with a polynomial second first integral, see for instance [13, 30]. Using the Ziglin's theory, Ito [17] provided necessary conditions (but not sufficient) for the integrability of the Henon-Heiles system (2). Thus he proved that necessary conditions for the integrability of system (2) are $A=6 B, A=B, A=2 B$. We know now that only the first two cases are integrable. The necessary condition for the integrability is important because using Theorem 2, we can prove that there cannot exist any second first integral of class $\mathcal{C}^{1}$ for the values of the parameters $A$ and $B$ for which the periodic orbits of Theorem 1 exist. Note that Ziglin's and Ito's results are on the existence or non-existence of analytic or meromorphic integrability in the sense of Liouville-Arnol'd, while our results are on the non-existence of integrability in the sense of Liouville-Arnol'd for any second first integral of class $\mathcal{C}^{1}$.

In some sense the Ziglin's theory is a continuation of Kovalevskaya's ideas used in the study of the first integrals of the rigid body with a fixed point, because it relates the non-integrability of the considered system with the behavior of some of its solutions as function of the complex time using the monodromy group of their variational equations. Ziglin's theory was extended to the so-called Morales-Ramis' theory, which replace the study of the monodromy group of the variational equations by the study of their Galois differential group, easier to analyze. See for more details [24] and the references quoted there. But as Ziglin's theory the Morales-Ramis' theory only can study the non-existence of meromorphic first integrals.

Kovalevskaya's idea and consequently Ziglin's and Morales-Ramis' theory go back to Poincaré (see Arnol'd [3]), who used the multipliers of the monodromy group of the variational equations associated to periodic orbits for studying the non-integrability of the differential equations. Poincaré's method allows to prove under convenient assumptions that the non Liouville-Arnol'd integrable systems have not any second first integral of class $\mathcal{C}^{1}$. See section 4 for more details. The main difficulty for applying Poincaré's non-integrability method to a given Hamiltonian system is to find for such a system periodic orbits having multipliers different from 1.

It seems that this result of Poincaré was forgotten by the mathematical community until modern Russian mathematicians (especially Kozlov) have recently published on it, see $[3,18]$. Here we will apply the Poincare criterion to the motion of the generalized Hénon-Heiles systems (2), and we will show that its motion is integrable and the two constants of motion have dependent gradients along the periodic orbits found in Theorem 1, or it is not Liouville-Arnol'd integrable with any second first integral of class $\mathcal{C}^{1}$. Of course for applying the Poincaré non-integrability theory to the HénonHeiles systems, we need to study some of the periodic orbits of these systems and to compute their multipliers. For doing that we shall use the averaging theory, see section 2 . 
On the other hand using the Melnikov integral, Holmes in [16] studied the nonintegrability of the systems (2) in a neighborhood of $A=B=-1$. Our proof on the non Liouville-Arnol'd integrability uses isolated periodic orbits in the energy levels, the Holmes' proof uses transverse homoclinic orbits. It is important to remark that both our method and Holmes method work for Hamiltonian systems which are close to integrable systems, but as we will see after a rescaling of the variables all systems (2) are close to integrable ones. The proof of Theorem 2 uses Theorem 1, and we obtain this last theorem perturbing an integrable Hamiltonian system, more precisely perturbing the harmonic oscillator.

Other interesting criterion on non-integrability also related with a Poincare's result was used by Meletlidou and Ichtiarouglou [21, 22, 23]. They consider perturbed Hamiltonian systems of the form $H=H_{0}+\varepsilon H_{1}$, where $H_{0}$ is a non-degenerate integrable Hamiltonian, and they show that some properties of the average value of the perturbing function $H_{1}$, evaluated along the non-isolated periodic orbits of $H_{0}$, are strongly connected with the non-integrability of the perturbed system. However this criterion cannot be applied to the Hénon-Heiles Hamiltonian (1) because it is degenerated.

As far as we know the first rigorous proof of non-existence of any first integral, not only meromorphic, for most of the values of the parameters in generalized Hénon-Heiles systems was given by Ragazzo [28]. He showed the existence of transversal homoclinic orbits to some periodic orbits of these systems, which have saddle-center equilibrium points with a homoclinic orbit. Yagasaky [29] extended this study to systems which are not a small perturbation of an integrable system.

In the remarkable paper [2] the authors link the Hénon-Heiles integrable case $A-$ $6 B=0$ with the Kovalevskaya top and the Manakov geodesic flow on $\mathrm{SO}(4)$. They provide a method to produce an one-dimensional family of birational maps between the Kowalewskaya and Hénon-Heiles invariant tori, and those of the Manakov flow. In this way our result could be extended to study the non Liouville-Arnol'd integrability of the rigid body motion with a fixed point.

The non-integrability of the case $A-2 B=0$ was conjectured numerically, but as far as we know, a rigorous proof of this fact was unsuccessful for a long time. Conte, Fordy and Pickering [8] proved the non-integrability in the sense of Painlevé of this case, and finally Morales-Ruiz, Ramis and Simó [25] were able to give a proof of the non-integrability by means of the study of the Galois differential group and the LaméHermite approach. But the non-integrable case $A-2 B=0$ is in the boundary of the non-integrable systems in the parameter space, where we cannot say anything. The classical Hénon-Heiles system is not Liouville-Arnol'd integrable.

The open regions of Figures 1 and 2 are those where Theorem 2 can show that the non-integrability in the sense of Liouville-Arnol'd is for any second first integral of class $\mathcal{C}^{1}$, or the system is integrable but the gradients of the two first integrals are dependent along the periodic orbits explicitly found in Theorem 1. But Ito showed that the only Liuoville-Arnol'd integrable cases could be $A-6 B=0, A-B=0$, and $A-2 B=0$, then all the open regions of Figures 1 and 2 minus these three cases are non-integrable with any second first integral of class $\mathcal{C}^{1}$. The only case of the previous three that lies inside the open regions of Figures 1 and 2 is $A=B$. This case has a second first integral [9] given by

$$
C=3 p_{X} p_{Y} \pm \varepsilon Y\left(3 X^{2}+Y^{2}\right)+3 X Y,
$$


where the sign + is for $A=B>0$, and the sign - is for $A=B<0$. We show that when $A=B$, there is one periodic orbit coming from the third solution of Theorem 1 . However if we calculate the gradients of the Hamiltonian and the previous first integral $C$, we get that they are not independent, and so statement (a) of Theorem 2 holds. On the other hand although the case $A-2 B=0$ is actually non-integrable, we cannot say anything since it is at the boundary of the open regions of Figures 1 and 2 where Theorem 2 holds.

We also have similar results to Theorems 1 and 2 for the generalized Hénon-Heiles systems with three cubic homogeneous terms, providing three free parameters. The polar change of variables to the angle-action ones is still useful, but in this case the expressions become so complicated and huge that we decided not to publish these results.

\section{The AVERAGING THEORY OF FIRST AND SECOND ORDER}

In this section we recall the averaging theory of second order to find periodic orbits. The averaging theory up to third order specifically for studying periodic orbits was developed in [6], see this paper for additional details and for the proofs of the results stated in this section related with the averaging theory.

In this work we only need this theory up to second order. It is summarized as follows.

Theorem 4. Consider the differential system

$$
\dot{x}(t)=\varepsilon F_{1}(t, x)+\varepsilon^{2} F_{2}(t, x)+\varepsilon^{3} R(t, x, \varepsilon),
$$

where $F_{1}, F_{2}: \mathbb{R} \times D \rightarrow \mathbb{R}^{n}, R: \mathbb{R} \times D \times\left(-\varepsilon_{f}, \varepsilon_{f}\right) \rightarrow \mathbb{R}^{n}$ are continuous functions, $T$ periodic in the first variable, and $D$ is an open subset of $\mathbb{R}^{n}$. Assume that the following hypothesis (i) and (ii) hold.

(i) $F_{1}(t, \cdot) \in C^{1}(D)$ for all $t \in \mathbb{R}, F_{1}, F_{2}, R$ and $D_{x} F_{1}$ are locally Lipschitz with respect to $x$, and $R$ is differentiable with respect to $\varepsilon$. We define $f_{1}, f_{2}: D \rightarrow \mathbb{R}^{n}$ as

$$
\begin{aligned}
& f_{1}(z)=\int_{0}^{T} F_{1}(s, z) d s \\
& f_{2}(z)=\int_{0}^{T}\left[D_{z} F_{1}(s, z) \int_{0}^{s} F_{1}(t, z) d t+F_{2}(s, z)\right] d s .
\end{aligned}
$$

(ii) For $V \subset D$ an open and bounded set and for each $\varepsilon \in\left(-\varepsilon_{f}, \varepsilon_{f}\right) \backslash\{0\}$, there exist $a \in V$ such that $f_{1}(a)+\varepsilon f_{2}(a)=0$ and $d_{B}\left(f_{1}+\varepsilon f_{2}\right) \neq 0$ (see its definition later on).

Then for $|\varepsilon|>0$ sufficiently small, there exists a $T$-periodic solution $\varphi(\cdot, \varepsilon)$ of the system such that $\varphi(0, \varepsilon) \rightarrow$ a when $\varepsilon \rightarrow 0$.

As usual we have denoted by $d_{B}\left(f_{1}+\varepsilon f_{2}\right)$, the Brouwer degree of the function $f_{1}+\varepsilon f_{2}: V \rightarrow \mathbb{R}^{n}$ at its fixed point $a$. A sufficient condition for showing that the Brouwer degree of a function $f$ at its fixed point $a$ is non-zero, is that the Jacobian of the function $f$ at $a$ (when it is defined) is non-zero.

If the function $f_{1}$ is not identically zero, then the zeros of $f_{1}+\varepsilon f_{2}$ are mainly the zeros of $f_{1}$ for $\varepsilon$ sufficiently small. In this case Theorem 4 provides the so-called averaging theory of first order. 
If the function $f_{1}$ is identically zero and $f_{2}$ is not identically zero, then the zeros of $f_{1}+\varepsilon f_{2}$ are the zeros of $f_{2}$. In this case Theorem 4 provides the so-called averaging theory of second order.

The method based on Theorem 4 consists essentially of finding $T$-periodic solutions for a differential system whose vector field depends on a small parameter $\epsilon$, by means of the averaging method. A quantitative relation between the solutions of some nonautonomous periodic differential system and the solutions of the averaged differential system, which is autonomous, is obtained. In this way a finite dimensional function related with the original problem is found, the simple zeros of this function correspond with the periodic orbits of the non-autonomous periodic differential system for values of the parameter $\varepsilon$ different from zero and sufficiently small. Here a simple zero $a$ of a function $f$ means that the Jacobian of $f$ at $a$ is not zero.

As we will see in the next section, after convenient changes of variables, we will be able to apply Theorem 4 to the Hénon-Heiles systems (2). This will allow to prove the existence of some periodic orbits for these systems and to provide the proof of Theorem 1.

\section{Proof of Theorem 1}

For proving Theorem 1 we shall apply Theorem 4 to the Hamiltonian system (2). Generically the periodic orbits of a Hamiltonian system with more than one degree of freedom are on cylinders fulfilled of periodic orbits. Therefore we cannot apply directly Theorem 4 to a Hamiltonian system, since the Jacobian of the function $f$ at the fixed point $a$ will be always zero. Then we must apply Theorem 4 to every Hamiltonian fixed level where the periodic orbits generically are isolated. Recall that the integrable Hamiltonian systems in the sense of Liouville-Arnol'd are non-generic, see [20].

On the other hand in order to apply Theorem 4 we need a small parameter $\varepsilon$. So in the Hamiltonian system (2) we change the variables $\left(x, y, p_{x}, p_{y}\right)$ to $\left(X, Y, p_{X}, p_{Y}\right)$ where $x=\varepsilon X, y=\varepsilon Y, p_{x}=\varepsilon p_{X}$ and $p_{y}=\varepsilon p_{Y}$. In the new variables, system (2) becomes

$$
\begin{aligned}
& \dot{X}=p_{X}, \\
& \dot{p}_{X}=-X-\varepsilon\left(A X^{2}+B Y^{2}\right), \\
& \dot{Y}=p_{Y}, \\
& \dot{p}_{Y}=-Y-2 \varepsilon B X Y .
\end{aligned}
$$

This system again is Hamiltonian with Hamiltonian

$$
\frac{1}{2}\left(p_{X}^{2}+p_{Y}^{2}+X^{2}+Y^{2}\right)+\varepsilon\left(B X Y^{2}+\frac{1}{3} A X^{3}\right) .
$$

As the change of variables is only a scale transformation, for all $\varepsilon$ different from zero, the original and the transformed systems (2) and (5) have essentially the same phase portrait, and additionally system (5) for $\varepsilon$ sufficiently small is close to an integrable one

First we change the Hamiltonian (6) and the equations of motion (5) to polar coordinates for $\varepsilon=0$, which is an harmonic oscillator. Thus we have

$$
X=r \cos \theta, \quad p_{X}=r \sin \theta, \quad Y=\rho \cos (\theta+\alpha), \quad p_{Y}=\rho \sin (\theta+\alpha) .
$$

Recall that this is a change of variables when $r>0$ and $\rho>0$. Moreover doing this change of variables appear in the system the angular variables $\theta$ and $\alpha$. Later on the 
variable $\theta$ will be used for obtaining the periodicity necessary for applying the averaging theory.

The fixed value of the energy in polar coordinates is

$$
h=\frac{1}{2}\left(r^{2}+\rho^{2}\right)+\varepsilon\left(\frac{1}{3} A r^{3} \cos ^{3} \theta+B r \rho^{2} \cos \theta \cos ^{2}(\theta+\alpha)\right),
$$

and the equations of motion are given by

$$
\begin{aligned}
\dot{r} & =-\varepsilon \sin \theta\left(A r^{2} \cos ^{2} \theta+B \rho^{2} \cos ^{2}(\theta+\alpha)\right), \\
\dot{\theta} & =-1-\varepsilon \cos \theta\left(A r \cos ^{2} \theta+\frac{\rho^{2}}{r} B \cos ^{2}(\theta+\alpha)\right), \\
\dot{\rho} & =-\varepsilon B r \rho \cos \theta \sin (2(\theta+\alpha)), \\
\dot{\alpha} & =\varepsilon \frac{\cos \theta}{r}\left(A r^{2} \cos ^{2} \theta+B\left(\rho^{2}-2 r^{2}\right) \cos ^{2}(\theta+\alpha)\right) .
\end{aligned}
$$

However the derivatives of the left hand side of these equations are with respect to the time variable $t$, which is not periodic. We change to the $\theta$ variable as the independent one, and we denote by a prime the derivative with respect to $\theta$. The angular variable $\alpha$ can not be used as the independent variable since the new differential system would not have the form (4) for applying Theorem 4. The system (8) goes over to

$$
\begin{aligned}
r^{\prime} & =\frac{\varepsilon r \sin \theta\left(A r^{2} \cos ^{2} \theta+B \rho^{2} \cos ^{2}(\theta+\alpha)\right)}{r+\varepsilon\left(A r^{2} \cos ^{3} \theta+B \rho^{2} \cos \theta \cos ^{2}(\theta+\alpha)\right)}, \\
\rho^{\prime} & =\frac{\varepsilon B r^{2} \rho \cos \theta \sin (2(\theta+\alpha))}{r+\varepsilon\left(A r^{2} \cos ^{3} \theta+B \rho^{2} \cos \theta \cos ^{2}(\theta+\alpha)\right)}, \\
\alpha^{\prime} & =-\frac{\varepsilon \cos \theta\left(B\left(\rho^{2}-2 r^{2}\right) \cos ^{2}(\theta+\alpha)+A r^{2} \cos ^{2} \theta\right)}{r+\varepsilon\left(B \rho^{2} \cos \theta \cos ^{2}(\theta+\alpha)+A r^{2} \cos ^{3} \theta\right)} .
\end{aligned}
$$

Of course this system has now only three equations because we do not need the $\theta$ equation. If we write the previous system as a Taylor series in powers of $\varepsilon$, we have

$$
\begin{aligned}
r^{\prime}= & \varepsilon \sin \theta\left(A r^{2} \cos ^{2} \theta+B \rho^{2} \cos ^{2}(\theta+\alpha)\right)- \\
& \varepsilon^{2} \frac{\sin 2 \theta}{8 r}\left(A r^{2}(1+\cos (2 \theta))+B \rho^{2}(1+\cos (2(\theta+\alpha)))^{2}+O\left(\varepsilon^{3}\right),\right. \\
\rho^{\prime}= & \varepsilon B r \rho \cos \theta \sin (2(\theta+\alpha))- \\
& \varepsilon^{2} B \rho \cos ^{2} \theta \sin (2(\theta+\alpha))\left(A r^{2} \cos ^{2} \theta+B \rho^{2} \cos ^{2}(2(\theta+\alpha))\right)+O\left(\varepsilon^{3}\right), \\
\alpha^{\prime}= & -\varepsilon \frac{\cos \theta}{r}\left(A r^{2} \cos ^{2} \theta+B\left(\rho^{2}-2 r^{2}\right) \cos ^{2}(\theta+\alpha)\right)+ \\
& \varepsilon^{2} \frac{\cos ^{2} \theta}{r^{2}}\left(A r^{2} \cos ^{2} \theta+B \rho^{2} \cos ^{2}(\theta+\alpha)\right) \\
& \left(A r^{2} \cos ^{2} \theta+B\left(\rho^{2}-2 r^{2}\right) \cos ^{2}(\theta+\alpha)\right)+O\left(\varepsilon^{3}\right) .
\end{aligned}
$$

Now system (9) is $2 \pi$-periodic in the variable $\theta$. In order to apply Theorem 4 we must fix the value of the first integral at $h>0$, and by solving equation (7) for $\rho$ we obtain

$$
\rho=\sqrt{\frac{h-r^{2} / 2-\varepsilon A r^{3} \cos ^{3} \theta / 3}{1 / 2+\varepsilon B r \cos \theta \cos ^{2}(\theta+\alpha)}} .
$$


Then substituting $\rho$ in equations (9), we obtain the two differential equations

$$
\begin{aligned}
r^{\prime}= & \varepsilon \sin \theta\left(A r^{2} \cos ^{2} \theta+B\left(2 h-r^{2}\right) \cos ^{2}(\theta+\alpha)\right)- \\
& \varepsilon^{2}\left(\frac{\sin 2 \theta}{8 r}\left(A r^{2}(1+\cos (2 \theta))+B\left(2 h-r^{2}\right)(1+\cos (2(\theta+\alpha)))\right)^{2}+\right. \\
& \frac{2}{3} A B r^{3} \sin \theta \cos ^{3} \theta \cos ^{2}(\theta+\alpha)+ \\
& \left.2 B^{2} h r \sin (2 \theta) \cos ^{4}(\theta+\alpha)-B^{2} r^{3} \sin (2 \theta) \cos ^{4}(\theta+\alpha)\right)+O\left(\varepsilon^{3}\right), \\
\alpha^{\prime}= & \varepsilon\left(\frac{B}{r}\left(3 r^{2}-2 h\right) \cos \theta \cos ^{2}(\theta+\alpha)-A r \cos ^{3} \theta\right)+ \\
& \varepsilon^{2}\left(A^{2} r^{2} \cos ^{6} \theta+\frac{2}{3} A B\left(6 h-5 r^{2}\right) \cos ^{4} \theta \cos ^{2}(\theta+\alpha)+\right. \\
& \left.\frac{B^{2}}{r^{2}}\left(r^{2}-2 h\right)^{2} \cos ^{2} \theta \cos ^{4}(\theta+\alpha)\right)+O\left(\varepsilon^{3}\right) .
\end{aligned}
$$

Clearly system (11) satisfies the assumptions of Theorem 4, and it has the form (4) with $F_{1}=\left(F_{11}, F_{12}\right)$ and $F_{2}=\left(F_{21}, F_{22}\right)$, where

$$
\begin{aligned}
& F_{11}=\sin \theta\left(A r^{2} \cos ^{2} \theta+B\left(2 h-r^{2}\right) \cos ^{2}(\theta+\alpha)\right), \\
& F_{12}=\frac{B}{r}\left(3 r^{2}-2 h\right) \cos \theta \cos ^{2}(\theta+\alpha)-A r \cos ^{3} \theta,
\end{aligned}
$$

and

$$
\begin{aligned}
F_{21}= & -\frac{\sin 2 \theta}{8 r}\left(A r^{2}(1+\cos (2 \theta))+B\left(2 h-r^{2}\right)(1+\cos (2(\theta+\alpha)))\right)^{2}- \\
& \frac{2}{3} A B r^{3} \sin \theta \cos ^{3} \theta \cos ^{2}(\theta+\alpha)-2 B^{2} h r \sin (2 \theta) \cos ^{4}(\theta+\alpha)+ \\
& B^{2} r^{3} \sin (2 \theta) \cos ^{4}(\theta+\alpha), \\
F_{22}= & A^{2} r^{2} \cos ^{6} \theta+\frac{2}{3} A B\left(6 h-5 r^{2}\right) \cos ^{4} \theta \cos ^{2}(\theta+\alpha)+ \\
& \frac{B^{2}}{r^{2}}\left(r^{2}-2 h\right)^{2} \cos ^{2} \theta \cos ^{4}(\theta+\alpha) .
\end{aligned}
$$

As $r \neq 0$ the functions $F_{1}$ and $F_{2}$ are analytical. Furthermore they are $2 \pi$-periodic in the variable $\theta$, the independent variable of system (11). However the averaging theory of first order does not apply because the average functions of $F_{1}$ and $F_{2}$ in the period vanish

$$
f_{1}(r, \alpha)=\int_{0}^{2 \pi}\left(F_{11}, F_{12}\right) d \theta=(0,0) .
$$

As the function $f_{1}$ of Theorem 4 is zero, we procede to calculate the function $f_{2}$ by applying the second order averaging theory. We have that $f_{2}$ is defined by

$$
f_{2}(r, \alpha)=\int_{0}^{2 \pi}\left[D_{r \alpha} F_{1}(\theta, r, \alpha) \cdot y_{1}(\theta, r, \alpha)+F_{2}(\theta, r, \alpha)\right] d \theta,
$$

where

$$
y_{1}(\theta, r, \alpha)=\int_{0}^{\theta} F_{1}(t, r, \alpha) d t
$$

The two components of the vector $y_{1}$ are

$$
\begin{aligned}
y_{11}= & \int_{0}^{\theta} F_{11}(t, r, \alpha) d t= \\
& \frac{1}{3}\left(B\left(2 h-r^{2}\right) \sin ^{2}(\theta / 2)(\cos (2(\theta+\alpha))+2 \cos (2 \alpha+\theta)+3)-A r^{2}\left(\cos ^{3} \theta-1\right)\right),
\end{aligned}
$$


and

$$
\begin{aligned}
y_{12}= & \int_{0}^{\theta} F_{12}(t, r, \alpha) d t= \\
& -\frac{A r}{12}(9 \sin \theta+\sin 3 \theta)-\frac{B h}{6 r}(3 \sin (2 \alpha+\theta)+\sin (2 \alpha+3 \theta)-4 \sin 2 \alpha+6 \sin \theta)+ \\
& \frac{B r}{4}(3 \sin (2 \alpha+\theta)+\sin (2 \alpha+3 \theta)-4 \sin (2 \alpha)+6 \sin \theta) .
\end{aligned}
$$

For the Jacobian matrix

we obtain

$$
D_{r \alpha} F_{1}(\theta, r, \alpha)=\left(\begin{array}{cc}
\frac{\partial F_{11}}{\partial r} & \frac{\partial F_{11}}{\partial \alpha} \\
\frac{\partial F_{12}}{\partial r} & \frac{\partial F_{12}}{\partial \alpha}
\end{array}\right),
$$

$$
\left(\begin{array}{ll}
\left(2 A r \cos ^{2} \theta-2 B r \cos ^{2}(\theta+\alpha)\right) \sin \theta & -2 B\left(2 h-r^{2}\right) \cos (\theta+\alpha) \sin \theta \sin (\theta+\alpha) \\
-A \cos ^{3} \theta+6 B \cos ^{2}(\theta+\alpha) \cos \theta & -\frac{2 B}{r}\left(3 r^{2}-2 h\right) \cos \theta \cos (\theta+\alpha) \sin (\theta+\alpha) \\
-\frac{B}{r^{2}}\left(3 r^{2}-2 h\right) \cos ^{2}(\theta+\alpha) \cos \theta &
\end{array}\right) .
$$

We can now calculate from Theorem 4 the function (12) and we obtain

$$
\begin{aligned}
f_{2}= & \left(-\frac{B r}{12}(6 B-A)\left(r^{2}-2 h\right) \sin 2 \alpha,\right. \\
& \left.\frac{1}{12}\left(r^{2}\left(5 A^{2}-12 A B-3 B^{2}\right)-2 B(A-6 B)\left(h-r^{2}\right) \cos (2 \alpha)+2 B h(6 A-B)\right)\right) .
\end{aligned}
$$

We have to find the zeros $\left(r^{*}, \alpha^{*}\right)$ of $f_{2}(r, \alpha)$, and to check that the Jacobian determinant

$$
\left|D_{r, \alpha} f_{2}\left(r^{*}, \alpha^{*}\right)\right| \neq 0
$$

Solving the equation $f_{2}(r, \alpha)=0$ we obtain five solutions $\left(r^{*}, \alpha^{*}\right)$ with $r^{*}>0$, namely

(14) $\left(\sqrt{2} h, \pm \operatorname{arcsec} \frac{B(A-6 B)}{4 B^{2}+6 A B-5 A^{2}}\right),\left(\sqrt{\frac{2 B h}{3 B-A}}, 0\right),\left(\sqrt{\frac{14 B h}{9 B-5 A}}, \pm \pi / 2\right)$.

The first two solutions are not good, because for them we get from (10) that $\rho=0$ when $\varepsilon=0$, and $\rho$ must be positive. The third solution exists if $B(3 B-A)>0$. The last two solutions exist if $B(9 B-5 A)>0$. The Jacobian (13) of the third solution is

$$
-\frac{5 B^{2} h^{2}(A-6 B)(A-2 B)(A+B)}{9(A-3 B)},
$$

and for the last two solutions the Jacobian coincides and is equal to

$$
\frac{7 B^{2} h^{2}(A-6 B)(5 A-2 B)(A-B)}{9(5 A-9 B)} .
$$

Summarizing, from Theorem 4 the third solution of $f_{2}(r, \alpha)=0$ provides a periodic orbit of system (11) (and consequently of the Hamiltonian system (5) on the Hamiltonian level $h>0)$ if $B(3 B-A)>0,(A-6 B)(A-2 B)(A+B) \neq 0$, and from (10) we get $\rho=\sqrt{2(A-2 B) h /(A-3 B)}$, we also need $(2 B-A)(3 B-A)>0$. The conditions $B(3 B-A)>0$ and $(2 B-A)(3 B-A)>0$ can be reduced to $B(2 B-A)>0$, where 
$(A-6 B)(A-2 B) \neq 0$ is included, but $A+B \neq 0$ is not. Then the third solution provides a periodic orbit when $B(2 B-A)>0$ and $A+B \neq 0$.

In a similar way the last two solutions of $f_{2}(r, \alpha)=0$ provide two periodic orbits of system (11) if $B(9 B-5 A)>0,(A-6 B)(5 A-2 B)(A-B) \neq 0$, and from (10) we get $\rho=\sqrt{2(5 A-2 B) h /(5 A-9 B)}$, we also need $(2 B-5 A)(9 B-5 A)>0$. The conditions $B(9 B-5 A)>0$ and $(2 B-5 A)(9 B-5 A)>0$ can be reduced to $B(2 B-5 A)>0$, where the condition $(A-6 B)(5 A-2 B)(A-B) \neq 0$ is included. Then the fourth and fifth solutions provide two periodic orbits whenever $B(2 B-5 A)>0$.

There is one periodic orbit if the third solution exists, and the last two solutions do not. There are two periodic orbits if the two last solutions exist, and not the third one, i.e. when $A+B=0$. Finally there are three periodic orbits if the third, fourth and fifth solutions exist. Now the statements of Theorem 1 follow easily.

The regions in the parameter space where periodic orbits exist are summarized in Figures 1 and 2.

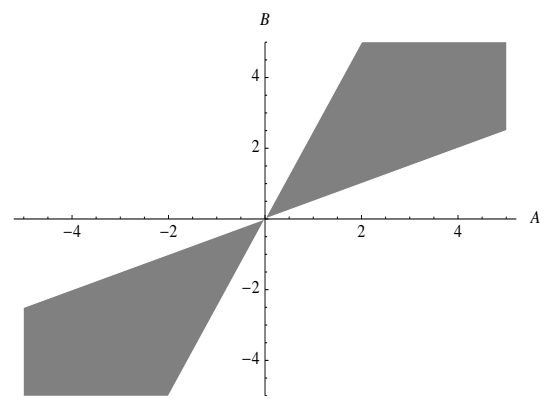

Figure 1. Open region $(2 B-5 A)(2 B-A)<0$ in the parameter space $(A, B)$ where there is at least one periodic orbit with multipliers different from 1 .

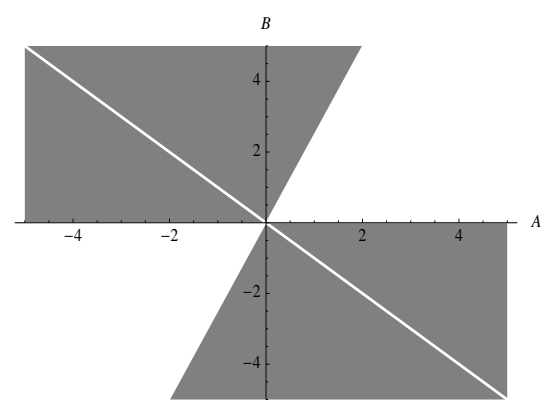

Figure 2. Open region $B(2 B-5 A)>0$ and $A+B \neq 0$ in the parameter space $(A, B)$ where there are at least three periodic orbits with multipliers different from 1 . When $A+B=0$, there are at least two periodic orbits with multipliers different from 1.

In the next section we will use the existence of these periodic orbits with multipliers different from 1 to study the non-integrability of the Hamiltonian system (5). 
As we mentioned in the introduction, Ragazzo [28] and Yagasaky [29] studied the non-integrability in two degrees of freedom Hamiltonian systems which have saddlecenters with homoclinic orbits. In fact, besides the equilibrium point center type at the origin, there are other equilibrium points $\left(X, p_{X}, Y, p_{Y}\right)$ for certain values of the parameters $A$ and $B$. They are

$$
\left(-\frac{1}{\varepsilon A}, 0,0,0\right) \text { and }\left(-\frac{1}{2 \varepsilon B}, 0, \pm \frac{\sqrt{2-A / B}}{2 \varepsilon B}, 0\right)
$$

when $\varepsilon \neq 0$. These two equilibrium points are saddle-center type and they can provide other way to show the non-integrability of the Hamiltonian system (5).

\section{Periodic orbits and the Liouville-Arnol'D integrability}

In this section we summarize some facts on the Liouville-Arnol'd integrability of the Hamiltonian systems, and on the theory of the periodic orbits of the differential equations, for more details see [1,4] and the subsection 7.1.2 of [4], respectively. We present these results for Hamiltonian systems of two degrees of freedom, because we are studying a Hamiltonian system with two degrees of freedom associated to the motion of generalized Hénon-Heiles systems, but these results work for an arbitrary number of degrees of freedom.

We recall that a Hamiltonian system with Hamiltonian $H$ of two degrees of freedom is integrable in the sense of Liouville-Arnol'd if it has a first integral $C$ independent with $H$ (i.e. the gradient vectors of $H$ and $C$ are independent in all the points of the phase space except perhaps in a set of zero Lebesgue measure), and in involution with $H$ (i.e. the parenthesis of Poisson of $H$ and $C$ is zero). For Hamiltonian systems with two degrees of freedom the involution condition is redundant, because the fact that $C$ is a first integral of the Hamiltonian system, implies that the mentioned Poisson parenthesis is always zero. A flow defined on a subspace of the phase space is complete if its solutions are defined for all time.

Now we shall state the Liouville-Arnol'd Theorem restricted to Hamiltonian systems of two degrees of freedom.

Theorem 5. Suppose that a Hamiltonian system with two degrees of freedom defined on the phase space $M$ has its Hamiltonian $H$ and the function $C$ as two independent first integrals in involution. If $I_{h c}=\{p \in M: H(p)=h$ and $C(p)=c\} \neq \emptyset$ and $(h, c)$ is a regular value of the map $(H, C)$, then the following statements hold.

(a) $I_{h c}$ is a two dimensional submanifold of $M$ invariant under the flow of the Hamiltonian system.

(b) If the flow on a connected component $I_{h c}^{*}$ of $I_{h c}$ is complete, then $I_{h c}^{*}$ is diffeomorphic either to the torus $\mathbb{S}^{1} \times \mathbb{S}^{1}$, or to the cylinder $\mathbb{S}^{1} \times \mathbb{R}$, or to the plane $\mathbb{R}^{2}$. If $I_{h c}^{*}$ is compact, then the flow on it is always complete and $I_{h c}^{*} \approx \mathbb{S}^{1} \times \mathbb{S}^{1}$.

(c) Under the hypothesis (b) the flow on $I_{h c}^{*}$ is conjugated to a linear flow on $\mathbb{S}^{1} \times \mathbb{S}^{1}$, on $\mathbb{S}^{1} \times \mathbb{R}$, or on $\mathbb{R}^{2}$.

The main result of this theorem is that the connected components of the invariant sets associated with the two independent first integrals in involution are generically submanifolds of the phase space, and if the flow on them is complete then they are diffeomorphic to a torus, a cylinder or a plane, where the flow is conjugated to a linear one. 
Using the notation of Theorem 5 when a connected component $I_{h c}^{*}$ is diffeomorphic to a torus, either all orbits on this torus are periodic if the rotation number associated to this torus is rational, or they are quasi-periodic (i.e. every orbit is dense in the torus) if the rotation number associated to this torus is not rational.

We consider the autonomous differential system

$$
\dot{x}=f(x),
$$

where $f: U \rightarrow \mathbb{R}^{n}$ is $C^{2}, U$ is an open subset of $\mathbb{R}^{n}$ and the dot denotes the derivative respect to the time $t$. We write its general solution as $\phi\left(t, x_{0}\right)$ with $\phi\left(0, x_{0}\right)=x_{0} \in U$ and $t$ belonging to its maximal interval of definition.

We say that $\phi\left(t, x_{0}\right)$ is $T$-periodic with $T>0$ if and only if $\phi\left(T, x_{0}\right)=x_{0}$ and $\phi\left(t, x_{0}\right) \neq x_{0}$ for $t \in(0, T)$. The periodic orbit associated to the periodic solution $\phi\left(t, x_{0}\right)$ is $\gamma=\left\{\phi\left(t, x_{0}\right), t \in[0, T]\right\}$. The variational equation associated to the $T$ periodic solution $\phi\left(t, x_{0}\right)$ is

$$
\dot{M}=\left(\left.\frac{\partial f(x)}{\partial x}\right|_{x=\phi\left(t, x_{0}\right)}\right) M
$$

where $M$ is an $n \times n$ matrix. The monodromy matrix associated to the $T$-periodic solution $\phi\left(t, x_{0}\right)$ is the solution $M\left(T, x_{0}\right)$ of $(18)$ satisfying that $M\left(0, x_{0}\right)$ is the identity matrix. The eigenvalues $\lambda$ of the monodromy matrix associated to the periodic solution $\phi\left(t, x_{0}\right)$ are called the multipliers of the periodic orbit.

For an autonomous differential system, one of the multipliers is always 1 , and its corresponding eigenvector is tangent to the periodic orbit.

A periodic solution of an autonomous Hamiltonian system always has two multipliers equal to one. One multiplier is 1 because the Hamiltonian system is autonomous, and another is 1 due to the existence of the first integral given by the Hamiltonian.

Theorem 6. If a Hamiltonian system with two degrees of freedom and Hamiltonian $H$ is Liouville-Arnol'd integrable, and $C$ is a second first integral such that the gradients of $H$ and $C$ are linearly independent at each point of a periodic orbit of the system, then all the multipliers of this periodic orbit are equal to 1.

Theorem 6 is due to Poincaré [27], section 36. It gives us a tool to study the non Liouville-Arnol'd integrability, independently of the class of differentiability of the second first integral. The main problem for applying this theorem is to find periodic orbits having multipliers different from 1.

Proof of Theorem 2. We assume that we are under the assumptions of Theorem 1, and that one of the three founded periodic orbits corresponding to the third, fourth and fifth solutions of (14) exist. Their associated Jacobians (15) and (16) are different from 1 playing with the energy level $h$. Since these Jacobians are the product of the four multipliers of these periodic orbits with two of them always equal to 1 , the remainder two multipliers cannot be equal to 1 . Hence under the assumptions of Theorem 1, by Theorem 6, either the Hénon-Heiles systems cannot be Liouville-Arnol'd integrable with any second first integral $C$, or the system is Liouville-Arnol'd integrable and the differentials of $H$ and $C$ are linearly dependent on some points of these periodic orbits. Therefore the theorem is proved.

The only integrable case inside the regions where Theorem 2 holds is the case $A=B$ [17], which is contained in statement (a) of Theorem 1 . Thus the two first integrals $H$ 
and $C$ given in (3) are dependent on some points of the periodic orbit corresponding to the third solution of (14), as can be easily checked. Therefore Corollary 3 is proved.

\section{Conclusions}

We have provided two new tools. One for studying the periodic orbits of the Hamiltonian systems in their fixed Hamiltonian levels. Here we have applied it to the generalized Hénon-Heiles Hamiltonian system (2) with two degrees of freedom obtaining Theorem 1, but this tool can be applied to Hamiltonian systems with an arbitrary degrees of freedom.

The second tool allows to study the non-integrability in the sense of LiouvilleArnol'd of the Hamiltonian systems, for any second first integral of class $\mathcal{C}^{1}$. We have applied it to the generalized Hénon-Heiles Hamiltonian system (5) with two degrees of freedom obtaining Theorem 2 and Corollary 3, but again, this tool can be applied to Hamiltonian systems with an arbitrary number of degrees of freedom.

It is important to remark that these two tools are based in the study of the periodic orbits via the averaging method, and that this method needs a small parameter, which is easy to obtain if we study Hamiltonian systems near integrable ones in the sense of Liouville-Arnol'd. However, the scale transformation introduced in the section 3 does not change the topology of the system, thus these results are valid for $\varepsilon=1$. The two Hamiltonian systems (2) and (5) have qualitatively the same phase portrait.

\section{ACKNowledgments}

The first author is partially supported by the grants MEC/FEDER MTM 200803437, CIRIT 2009SGR 410 and ICREA Academia.

\section{REFERENCES}

[1] Abraham R. and Marsden J.E., Foundations of Mechanics, Benjamin, Reading, Masachusets, 1978.

[2] Adler M. and van Moerbeke P., The Kowalewski and Hénon-Heiles motions as Manakov geodesic flows on $\mathrm{SO}(4)$ - a two-dimensional family of Lax pairs, Comm. Math. Phys. 113 (1988), 659-700.

[3] Arnol'd V.I., Forgotten and neglected theories of Poincaré, Russian Math. Surveys 61 No. 1 (2006), $1-18$.

[4] Arnol'd V.I., Kozlov V. and Neishtadt A., Dynamical Systems III. Mathematical Aspects of Classical and Celestial Mechanics, Third Edition, Encyclopaedia of Mathematical Science, Springer, Berlin, 2006.

[5] Brack M., Orbits with analytical Scaling Constants in Hénon-Heiles type potentials, Fundations of Phys., 31 (2001), 209-232.

[6] Buică A. and Llibre J., Averaging methods for finding periodic orbits via Brouwer degree, Bull. Sci. Math. 128 (2004), 7-22.

[7] Chazy J., Sur les équations difféntielles du troisième ordre et d'ordre supérieur dont l'intégrale générale a ses points critiques fixes, Acta Mathematica, 34 (1911), 317-385.

[8] Conte R., Fordy A.P. and Pickering A. A perturbative Painlevé approach to nonlinear differential equations, Physica D, 69, (1993), 33-58.

[9] Conte R., Musette M. and Verhoeven C., Explicit integration of the Hénon-Heiles Hamiltonians, Journal of Nonlinear Mathematical Physics, 12, 1 (2005), 212-227.

[10] Churchill R., Pecelli G. and Rod D., in Stochastic Behaviour in Classical and Quantum Hamiltonian Systems, G. Casati and J. Ford eds., Springer NY 1979, 76-136.

[11] Davies K., Huston T. and Baranger M., Calculations of periodic trajectories for the Hénon-Heiles Hamiltonian using the monodromy method, Chaos 2, (1992) 215-224.

[12] ElSabaa F. and Sherief H., Periodic orbits of galactic motions, Astrophys. and Space Sci. 167, (1990) 305-315.

[13] Fordy A.P., The Hénon-Heiles system revisited, Physica D 52 (1991), 204-210. 
[14] Grammaticos B., Dorizzi B. and Padjen R., Painlevé property and integrals of motion for the Henon-Heiles system, Physics Letters 89A, (1982), 111-113.

[15] Hénon M. and Heiles C., The applicability of the third integral of motion: some numerical experiments, Astron. J. 69 (1964), 73-84.

[16] Holmes P., Proof of non-integrability for the Hénon-Heiles Hamiltonian near an exceptional integrable case, Physica D: Nonlinear Phenomena 5 (1982), 335-347.

[17] Ito H., Non-integrability of Hénon-Heiles system and a theorem of Ziglin, Kodai Math. J., 8 (1985), $120-138$.

[18] Kozlov V.V., Integrability and non-integrability in Hamiltonian mechanics, Russian Math. Surveys 38 No. 1 (1983), 1-76.

[19] Maciejewski A., Radzki W. and Rybicki S., Periodic trajectories near degenerate equilibria in the Hénon-Heiles and Yang-Mills Hamiltonian systems, J. Dyn. and Diff. Eq. 17 (2005), 475-488.

[20] Markus L. and Meyer, K.R., Generic Hamiltonian Dynamical Systems are neither integrable nor ergodic, Memoirs of the Amer. Math. Soc. 144, 1974.

[21] Meletlidou E. and Ichtiaroglou S., A criterion for non-integrability based on Poincaré's theorem, Physica D 71 (1994), 261-268.

[22] Meletlidou E. and Ichtiaroglou S., On the number of isolating integrals in perturbed Hamiltonian system with $n \geq 3$ degrees of freedom, J. Phys. A: Math. Gen. 27 (1994), 3919-3926.

[23] Meletlidou E., Stagika G. and Ichtiaroglou S., Non-integrability and structure of the resonance zones in a class of galactic potentials, Cel. Mech. and Dyn. Astron. 91 (2005), 323-335.

[24] Morales-Ruiz J.J., Differential Galois Theory and non-integrability of Hamiltonian systems, Progress in Math. Vol. 178, Birkhauser, Verlag, Basel, 1999.

[25] Morales-Ruiz J.J., Ramis J.P. and Simó C., Integrability of Hamiltonian Systems and Differential Galois Groups of Higher Variational Equations, Annales Scientifiques de l'École Normale Supérieure, 40, (2007), 845-884

[26] Ozaki J. and Kurosaki S., Periodic orbits of Hénon Heiles Hamiltonian Prog. in Theo. Phys. 95 (1996), 519-529.

[27] Poincaré H., Les méthodes nouvelles de la mécanique céleste, Vol. I, Gauthier-Villars, Paris 1899.

[28] Ragazzo C.G., Nonintegrability of Some Hamiltonian systems, Scattering and Analytic Continuation, Comm. Math. Phys. 166 (1994), 255-277.

[29] Yagasaky K., Horseshoes in Two-Degree-of-Freedom Hamiltonian Systems with Saddle-Centers, Arch. Rational Mech. Anal. 154 (2000) 275-296.

[30] Sarlet W., New aspects of integrability of generalized Hénon-Heiles systems, J. Phys. A: Math. Gen. 24 (1991), 5245-5251.

${ }^{1}$ Departament de Matemàtiques, Universitat Autònoma de Barcelona, 08193 Bellaterra, Barcelona, Catalonia, Spain

E-mail address: jllibre@mat.uab.cat

${ }^{2}$ Departamento de Física, Universidad Autónoma Metropolitana-Iztapalapa, P.O. Box 55-534, MÉxico, D.F., 09340 MÉxico

E-mail address: lidia@xanum.uam.mx 